\title{
WJ-MSCs intervention may relieve intrauterine adhesions in female rats via TGF-ß31-mediated Rho/ROCK signaling inhibition
}

\author{
JUN LI* ${ }^{*}$, BO HUANG ${ }^{*}$, LAN DONG ${ }^{*}$, YAJUAN ZHONG and ZHIXIN HUANG \\ Department of Obstetrics and Gynecology, Renmin Hospital of Wuhan University, Wuhan, Hubei 430060, P.R. China
}

Received June 26, 2019; Accepted March 31, 2020

DOI: $10.3892 / \mathrm{mmr} .2020 .11646$

\begin{abstract}
Estrogen is a commonly used hormone in the adjuvant treatment of intrauterine adhesion (IUA), which can promote endometrial growth. Stem cell transplantation has also been reported to promote endometrial regeneration in IUA due to its potential differentiative capacity. Human Wharton's jelly mesenchymal stem cells (WJ-MSCs) are isolated from the umbilical cord, possess strong self-renewal and proliferative abilities, and are hypo-immunogenic and non-tumorigenic. Therefore, the present study aimed to investigate the therapeutic effects and underlying mechanism of WJ-MSCs transplantation with estrogen treatment, separately or as a combined therapy, on IUA. The IUA model was established using the ethanol damage method. A total of 50 Sprague-Dawley female rats were randomly divided into the control, IUA model, WJ-MSCs treatment, estrogen treatment and WJ-MSCs+ estrogen treatment groups (n=10/group). WJ-MSCs were injected three times at 5-day intervals. IUA rats in the estrogen group received $0.2 \mathrm{mg} / \mathrm{kg}$ estrogen through intragastric administration, once every 2 days for 8 weeks. Morphological changes were evaluated by hematoxylin-eosin staining. Immunohistochemical evaluations of pan-keratin, vimentin, transforming growth factor (TGF)- $\beta 1$, RhoA, RhoB, RhoC, Rho-associated coiled-coil-containing protein kinase (ROCK)I, and ROCKII expression were performed in uterine tissue. After treatment, the uterine specimens were observed to have increased uterine thickness and gland numbers in all treatment groups compared with the IUA group; however, the degree of restoration in the independent WJ-MSCs and estrogen treatment groups was better than in the combined
\end{abstract}

Correspondence to: Dr Zhixin Huang, Department of Obstetrics and Gynecology, Renmin Hospital of Wuhan University, 99 Zhang Zhidong Road, Wuchang, Wuhan, Hubei 430060, P.R. China

E-mail: huanjiu472qiaohuai@163.com

*Contributed equally

Key words: intrauterine adhesion, Wharton's jelly mesenchymal stem cells, transforming growth factor- $\beta 1$, RhoA/ROCKI signaling pathway treatment group. Immunohistochemical analysis demonstrated that pan-keratin expression was increased, and RhoA, ROCKI and TGF- $\beta 1$ expression was significantly inhibited in the WJ-MSCs and WJ-MSCs + estrogen treatment groups compared with the IUA group; however, the expression levels of these proteins were similar among all treatment groups. No change in vimentin expression was detected in any treatment group. The expression levels of RhoB, RhoC and ROCKII were clearly not affected by WJ-MSCs intervention alone. In conclusion, transplantation of WJ-MSCs may repair endometrial damage in IUA rats via TGF- $\beta 1$-mediated inhibition of RhoA/ROCKI signaling.

\section{Introduction}

Intrauterine adhesion (IUA), also named Asherman's syndrome, is characterized by endometrial fibrosis and IUAs with partial or complete uterine cavity occlusion (1). IUA generally occurs following endometrial damage, non-pregnant uterine tract injury, miscarriage and infection (2). Hypomenorrhea, pelvic pain and infertility are the main clinical manifestations in patients with IUA (3). The primary objective of IUA treatment is to restore endometrial regeneration and the shape of the uterine cavity, and prevent recurrence of adhesion. Although hysteroscopic adhesiolysis is a standard therapy for IUA treatment, numerous adjuvant therapies are required due to the varying degrees of IUA (4).

Estrogen is a commonly used hormone therapy for IUA, which promotes endometrial growth during preoperative treatment and enhances endometrial regeneration during postoperative treatment (5). However, estrogen therapy as an adjuvant treatment commonly needs to be combined with other therapies to obtain maximal outcomes. In recent years, mesenchymal stem cell (MSC) or endometrial stem cell transplant into the uterine cavity of patients with IUA or female rats has been reported to promote endometrial regeneration (6), which may be due to the potential differentiative capacities of the stem cells (7).

Human Wharton's jelly MSCs (WJ-MSCs) isolated from the umbilical cord possess strong self-renewal and proliferative activities, are hypo-immunogenic and non-tumorigenic, and are not associated with ethical controversy (8). Commonly, WJ-MSCs are discriminated by the detection of positive cell makers, such as CD44 and CD105 (matrix receptors), CD73 
glycoprotein and CD90 (Thy1), and the lack of hematopoietic lineage markers (CD34 and CD45) (9). WJ-MSCs, which share the natural homing capabilities of MSCs, demonstrate a promising cell therapy in future clinical applications for degenerative diseases (9). It was previously reported that WJ-MSC transplantation prevented early pregnancy loss in a rat model of spontaneous abortion (10). However, to the best of our knowledge, research regarding the application of WJ-MSCs to treat IUA is rarely reported.

Endometrial fibrosis and endometrial regeneration disorders are key to the formation of IUA (11). Studies have reported that epithelial-mesenchymal transition is one possible pathogenic factor of IUA, and this process was associated with epithelial cell regeneration disorder following progression of endometrial basal layer injury to endometrial fibrosis $(12,13)$. Transforming growth factor (TGF) $\beta 1$ is the most important fiber-forming cytokine, and is also an upstream signaling factor for Rho, which is one of the most important members of the Rho GTPase family, which includes A, B and C isoforms (14). Rho-associated coiled-coil-containing protein kinase (ROCK) is the most studied downstream effector molecule of Rho (15). The Rho/ROCK signaling pathway mediated by TGF- $\beta 1$ could induce fibrotic lesions (16). Keratin and vimentin are members of the intermediate filament family and are implicated in regulating a variety of cellular functions, especially in epithelial cells $(17,18)$. Considering all these findings, the objective of the present study was to investigate the therapeutic effects of estrogen and WJ-MSCs, separately or as a combined therapy, for IUA, and to further explore whether a TGF- $\beta 1$-mediated Rho/ROCK pathway can function in IUA.

\section{Materials and methods}

Animals. Sprague-Dawley female rats (age, 9 weeks; weight $180-220 \mathrm{~g}$ ) were purchased from Shanghai SLAC Laboratory Animal Co., Ltd. All rats were housed under specific pathogen-free conditions with a 12-h day/night cycle, and ad libitum access to food and water. All animals were maintained at a temperature of $22 \pm 1^{\circ} \mathrm{C}$ and humidity of $55 \pm 5 \%$. All the experimental operations for rats conformed to the Guide for the Care and Use of Laboratory Animals (19). This study was approved by the ethics committee of the People's Hospital of Wuhan University. A total of 55 rats were used, of which five rats were used in the preliminary experiments, and 50 rats were used in the current study.

WJ-MSCs culture and identification. WJ-MSCs were acquired from Professor Chen Yantian (20) (Cell Culture and Bioprocess Engineering Lab, School of Pharmacy, Shanghai Jiao Tong University). The cells were cultured in complete medium, comprising $\alpha$-minimum essential medium (cat. no. 12571063; Gibco; Thermo Fisher Scientific, Inc.), $10 \%$ fetal bovine serum (FBS; cat. no. 16140071; Gibco; Thermo Fisher Scientific, Inc.) and 1\% penicillin/streptomycin (cat. no. B540734; Sangon Biotech Co., Ltd.), and maintained at $37^{\circ} \mathrm{C}$ in a humidified atmosphere of $95 \%$ air and $5 \% \mathrm{CO}_{2}$. The phenotype of WJ-MSCs was determined by flow cytometry (FACSCalibur; BD Biosciences). Flow cytometric analysis was conducted with fluorochrome-conjugated antibodies against human CD73, CD90, CD105, CD34 and CD43. Briefly, the cells in the logarithmic phase were suspended in Hank's Balanced Salt Solution (HBSS) supplemented with 2\% FBS, and the cell density was adjusted to $1 \times 107$ cells $/ \mathrm{ml}$. Then, cells (100 $\mu \mathrm{l})$ were transferred to a 1.5-ml Eppendorf tube with antibodies against CD73 (cat. no. 550257; BD Biosciences), CD90 (cat. no. 555596; BD Biosciences), CD105 (cat. no. 560839; BD Biosciences), CD34 (cat. no. 550761; BD Biosciences) and CD45 (cat. no. 555483; BD Biosciences) (20 $\mu \mathrm{l})$, and incubated on ice for $30 \mathrm{~min}$. After washing three times, cells were transferred to a flow tube and resuspended in HBSS for flow cytometry on a BD FACSCalibur flow cytometer (BD Biosciences). The data were analyzed using FlowJo 10 software (FlowJo LLC).

Establishment of a rat model of IUA. The IUA rat model was constructed using the ethanol damage method (21). Briefly, rats were anesthetized with $1 \%$ pentobarbital sodium $(30 \mathrm{mg} / \mathrm{kg})$ through intraperitoneal injection, and the abdominal wall and cavity was surgically opened to expose the uterus under sterile conditions. The upper and lower ends of the bilateral uterine walls were clamped with bulldog clamps and $0.5 \mathrm{ml}$ 95\% ethanol was injected into the bottom of the uterine wall. After maintaining it for $3 \mathrm{~min}$, saline solution was injected into the uterine wall twice for washing. In addition, saline solution was used to replace the ethanol injection in the control group following the same operation. Finally, the abdominal cavity and wall were closed in layers, and 80,000 U/100 mg penicillin (North China Pharmaceutical Co., Ltd) was intramuscularly injected to prevent infection 3 days after the operation. After 14 days, IUA was confirmed by evaluating the histopathological changes in the uterine specimens using hematoxylin and eosin (H\&E) staining.

Experimental protocol for IUA treatment. A total of 50 Sprague Dawley female rats were used in the present study. The rats were randomly divided into five groups ( $\mathrm{n}=10$ rats/group): Control group; IUA model group; WJ-MSCs treatment group; estrogen treatment group; and WJ-MSCs + estrogen treatment group. IUA model rats were prepared as aforementioned. In the WJ-MSCs treatment group, 2 weeks after IUA model construction, a relaparotomy was performed and $2 \times 10^{6} \mathrm{WJ}-\mathrm{MSCs}$ at middle passage (P3-P7) were injected into the left uterine horn of IUA rats. In total, the intraperitoneal injections of WJ-MSCs were conducted three times at 5-day intervals. In the estrogen treatment group, 2 weeks after model rats were successfully prepared, IUA rats received $0.2 \mathrm{mg} / \mathrm{kg}$ estrogen (cat. no. E8875; Sigma-Aldrich; Merck KGaA) through intragastric administration, once every 2 days for 8 weeks. In the WJ-MSCs + estrogen treatment group, 2 weeks after IUA model construction, model rats were treated with WJ-MSCs injection and estrogen intragastric administration together. During the period, the health and behavior of rats were observed every other day. After 8 weeks of intervention, the rats were anesthetized with $1 \%$ pentobarbital sodium $(30 \mathrm{mg} / \mathrm{kg})$ by intraperitoneal injection prior to sacrifice by decapitation (animal death was verified by observing no behavior after $5 \mathrm{~min}$ ), and the uterine tissues of the experimental rats were resected.

$H \& E$ staining. The pathology of the specimens in each group was evaluated by H\&E staining under an inverted microscope 
A

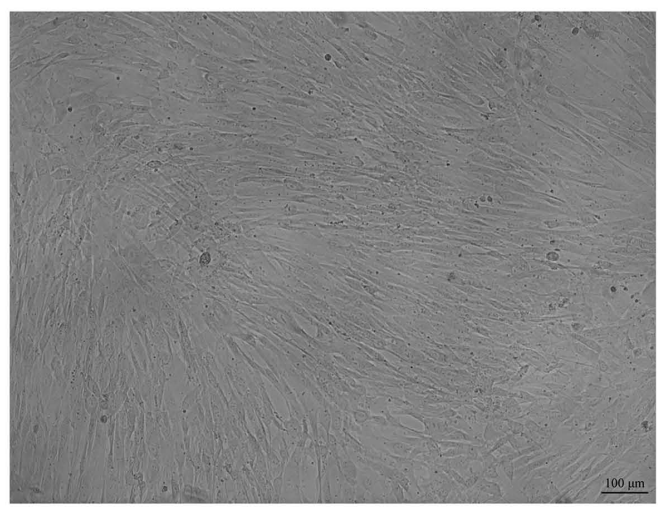

B
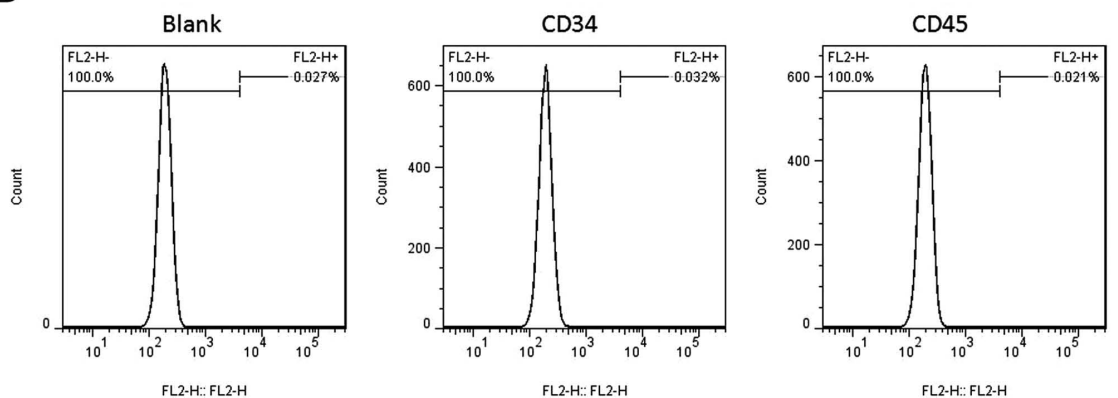

$\mathrm{CD} 73$
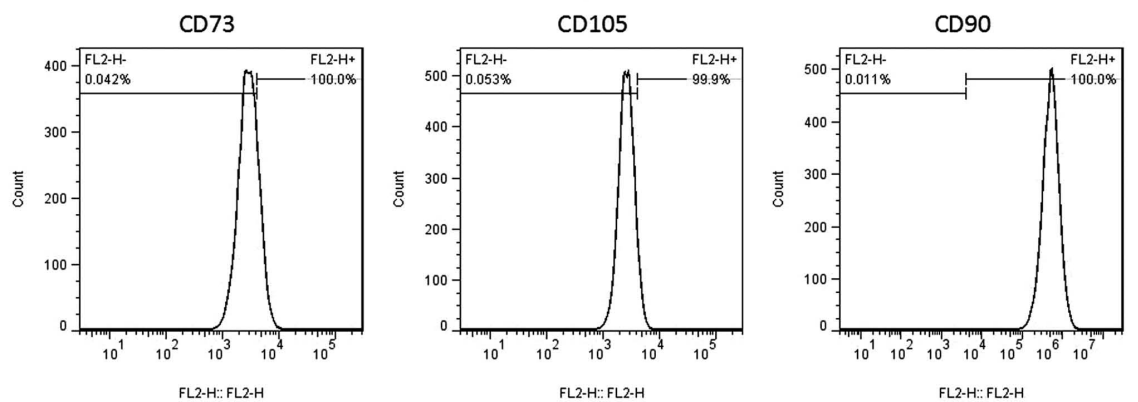

Figure 1. WJ-MSCs identification. (A) Morphological characteristics of WJ-MSCs (magnification, x100; scale bar, $100 \mu \mathrm{m}$ ). (B) Cell surface marker assessment of CD73, CD90, CD105, CD34 and CD43, as detected by flow cytometry. WJ-MSCs, Wharton's jelly mesenchymal stem cells.

(IX73; x40 magnification; Olympus Corporation). In brief, uterine tissues were fixed at room temperature for $48 \mathrm{~h}$ in $10 \%$ formalin and dehydrated in ascending concentrations of ethanol: $70 \%$ for $3 \mathrm{~h}, 80 \%$ for $40 \mathrm{~min}, 95 \%$ two times for $40 \mathrm{~min}$ each, $100 \%$ for $40 \mathrm{~min}$, and $100 \%$ again for $30 \mathrm{~min}$. Then, the dehydrated tissues were cleared by xylene and filtered into wax for $1 \mathrm{~h}$ three times consecutively. After embedding in paraffin, the specimens were cut into $4-\mu \mathrm{m}$ sections and dried at $60^{\circ} \mathrm{C}$ for $30 \mathrm{~min}$. Next, the slides were deparaffinized and rehydrated prior to staining with $\mathrm{H} \& \mathrm{E}$. The uterine thickness and the number of glands were observed under a light microscope (magnification, $\mathrm{x} 40$ ).

Immunohistochemistry. The expression levels of pan-keratin, vimentin, TGF- $\beta 1$, Rho subfamily members (RhoA, RhoB and RhoC), ROCKI and ROCKII in uterine tissue were examined using immunohistochemistry. After slide preparation as aforementioned, antigen retrieval was performed by inhibiting endogenous peroxidase by incubation with $3 \% \mathrm{H}_{2} \mathrm{O}_{2}$ for $10 \mathrm{~min}$ at room temperature, followed by microwave heating for $3 \mathrm{~min}$ to expose antigenic sites.
Non-specific binding was blocked with PBS containing $10 \%$ goat serum (Wuhan Boster Biological Technology, Ltd.) for $30 \mathrm{~min}$ at $37^{\circ} \mathrm{C}$ prior to transfer to an incubator (DHG-9053A; Shanghai Jinghong Experimental Equipment Co., Ltd.) for $30 \mathrm{~min}$ at $37^{\circ} \mathrm{C}$. The slides were incubated with primary antibodies against pan-keratin (1:10; cat. no. ab8068; Abcam), vimentin (1:500; cat. no. ab8069; Abcam), TGF- $\beta 1$ (1:100; cat. no. ab92486; Abcam), RhoA (1:1,000; cat. no. ab54835; Abcam), RhoB (1:500; cat. no. bs-11142R; BIOSS), RhoC (1:100; cat. no. ab180785; Abcam), ROCKI (1:100; cat. no. ab45171; Abcam), and ROCKII (1:200; cat. no. ab71598; Abcam) overnight at $4^{\circ} \mathrm{C}$. Then, the slides were incubated with a secondary antibody for $30 \mathrm{~min}$ at $37^{\circ} \mathrm{C}(1: 1,000$; cat. no. PV-9000; OriGene Technologies, Inc.) followed by washing with PBS three times ( $5 \mathrm{~min} /$ wash). Finally, the detection of the bound antibody was performed using the avidin-biotin complex reagent for $30 \mathrm{~min}$ at $37^{\circ} \mathrm{C}$. Diaminobenzidine solution was added and incubated for $2 \mathrm{~h}$ at room temperature with the slides, then the slides were counterstained with hematoxylin for $1 \mathrm{~min}$ at room temperature, rinsed with sterile water and air-dried. Finally, the slides were sealed 


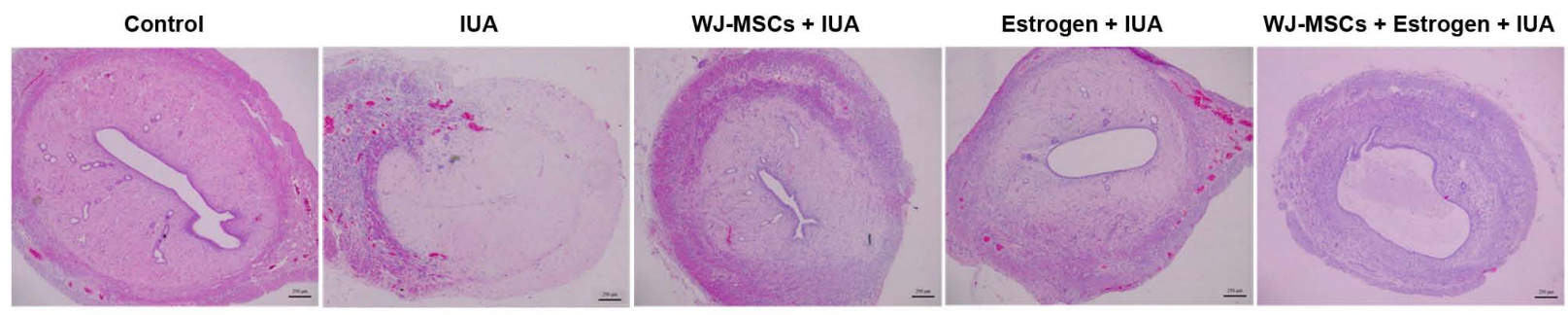

Figure 2. Histology of uterine endometrium for rats in each group, as detected by hematoxylin \& eosin staining (magnification, $\mathrm{x} 40$; scale bar, $250 \mu \mathrm{m}$ ). IUA, intrauterine adhesion; WJ-MSCs, Wharton's jelly mesenchymal stem cells.

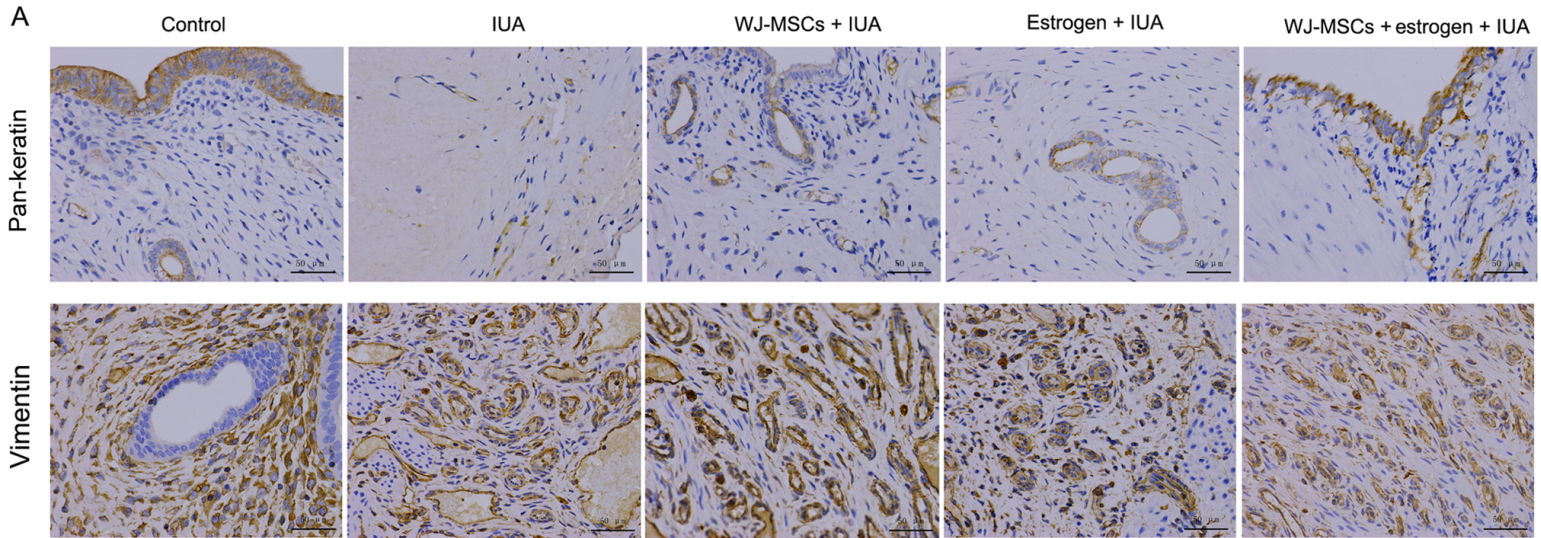

B
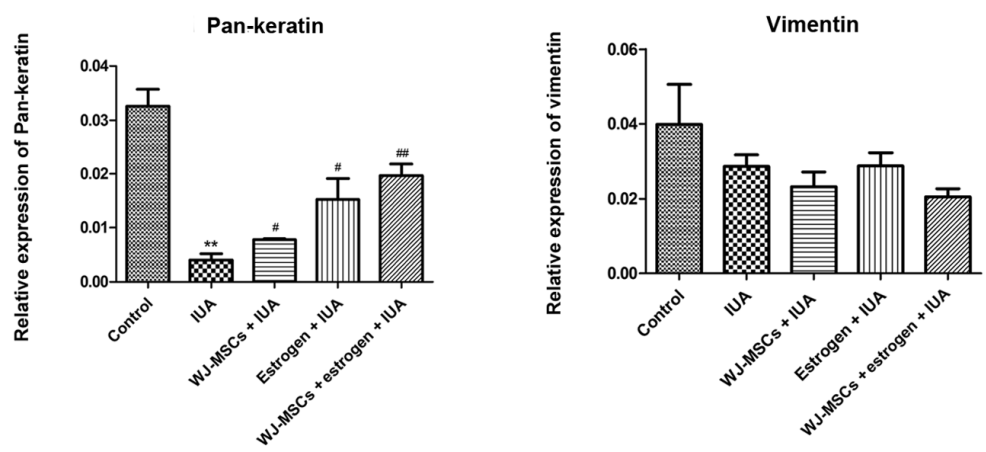

Figure 3. (A) Immunohistochemical staining, and (B) semi-quantitative analysis of immunohistochemical data for Pan-keratin and vimentin in each group. Scale bar, $50 \mu \mathrm{m}$. ${ }^{* *} \mathrm{P}<0.01$, compared with the control group; ${ }^{*} \mathrm{P}<0.05,{ }^{\# \prime} \mathrm{P}<0.01$ compared with the IUA model group. IUA, intrauterine adhesion; WJ-MSCs, Wharton's jelly mesenchymal stem cells.

with neutral resin and visualized under a fluorescent microscope (TE2000-E; Nikon Corporation). The expression of proteins was calculated based on the average gray value from six randomly selected fields at x400 magnification. Briefly, the area of positive staining and tissue area were analyzed by Image-Pro Plus 6.0 (Media Cybernetics, Inc.). Light yellow, brown-yellow or dark brown were regarded as positive staining. The percentage of positive expression was calculated using the following equation: Area of positive staining/ area of tissue.

Statistical analysis. All data are presented as the mean \pm standard deviation (SD). GraphPad Prism 5 (GraphPad Software, Inc.) was used for data analysis and graphics. All experiments were repeated three times. Statistical analyses were performed using one-way ANOVA, followed by Tukey's post hoc test. $\mathrm{P}<0.05$ was considered to indicate a statistically significant difference.

\section{Results}

WJ-MSCs identification by flow cytometry. The morphology of WJ-MSCs was observed as fusiform in shape (Fig. 1A). The cell surface markers (CD34, CD45, CD73, CD90 and CD105) were detected in WJ-MSCs by flow cytometry (Fig. 1B). As a result, WJ-MSCs presented positive expression of CD73, CD90 and CD105, and negative expression of CD34 and CD45. CD34 and CD45 are not markers for WJ-MSCs; therefore, this confirmed the cells were WJ-MSCs.

Histopathological assessment of uterine tissues. Histopathological changes in uterine specimens were evaluated by $H \& E$ staining. The results revealed that uterine thickness of the model rats was thinner and gland numbers were decreased compared with rats in the control group. In addition, the uterine orifice was closed. After WJ-MSCs or estrogen treatment, the uterine thickness of rats recovered to 

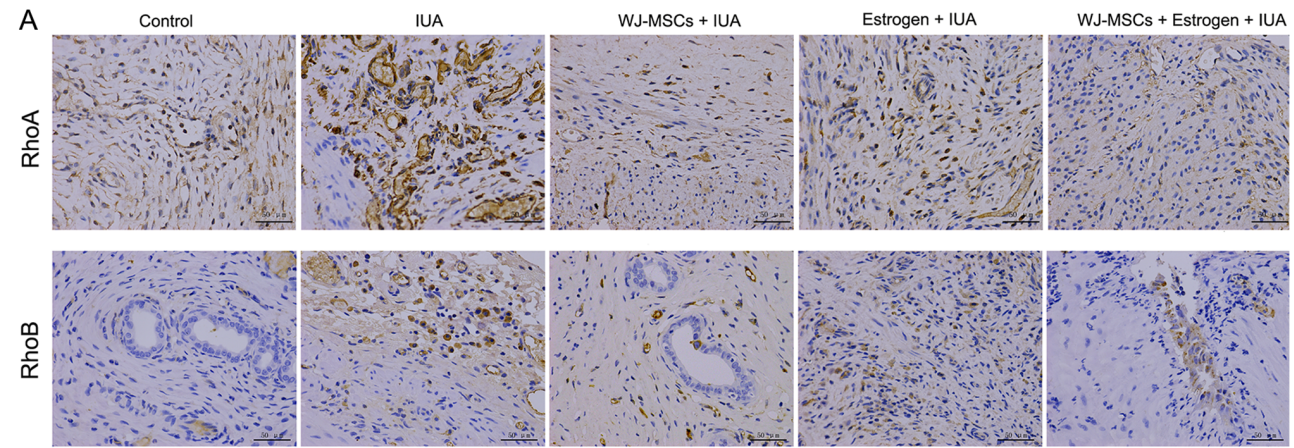

B

RhoA

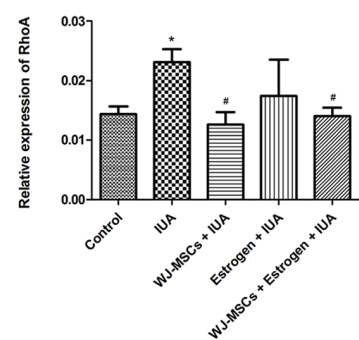

RhoB

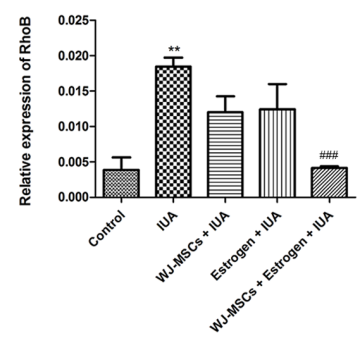

Figure 4. (A) Immunohistochemical staining, and (B) semi-quantitative analysis of immunohistochemical data for RhoA and RhoB in each group. Scale bar, $50 \mu \mathrm{m} .{ }^{*} \mathrm{P}<0.05,{ }^{* *} \mathrm{P}<0.01$ compared with the control group; ${ }^{\#} \mathrm{P}<0.05,{ }^{\# \# \#} \mathrm{P}<0.001$ compared with the model group. IUA, intrauterine adhesion; WJ-MSCs, Wharton's jelly mesenchymal stem cells.
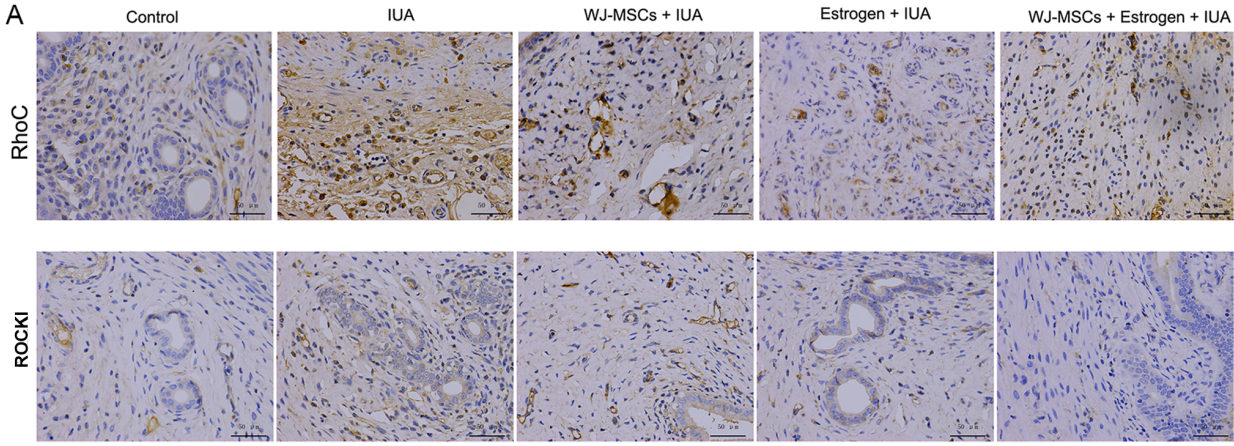

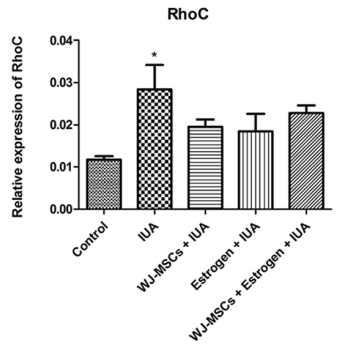

ROCKI

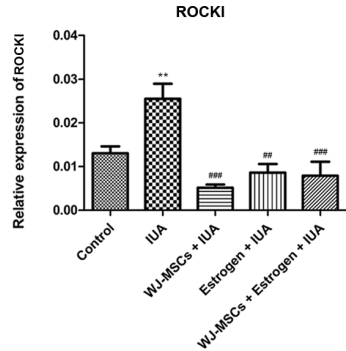

Figure 5. (A) Immunohistochemical staining, and (B) semi-quantitative analysis of immunohistochemical data for RhoC and ROCKI in each group. Scale bar, $50 \mu \mathrm{m} .{ }^{*} \mathrm{P}<0.05,{ }^{* *} \mathrm{P}<0.01$ compared with the control group; ${ }^{\# /} \mathrm{P}<0.01,{ }^{\# \# /} \mathrm{P}<0.001$ compared with the model group. IUA, intrauterine adhesion; ROCKI, Rho-associated coiled-coil-containing protein kinase I; WJ-MSCs, Wharton's jelly mesenchymal stem cells.

near normal, and the number of glands increased. The uterine tissue of rats in the WJ-MSCs + estrogen treatment group presented with increased uterine thickness and gland numbers, as well as an improvement in IUA symptoms; however, the degree of IUA restoration was less than in the WJ-MSCs or estrogen treatment groups (Fig. 2).

Immunohistochemistry. The expression levels of pan-keratin, vimentin, TGF- $\beta 1$, RhoA, RhoB, RhoC, ROCKI and ROCKII were detected by immunohistochemistry.In the IUA model group, the expression of pan-keratin was markedly reduced compared with the control group $(\mathrm{P}=0.0011)$, and its expression was gradually increased after WJ-MSCs $(\mathrm{P}=0.0386)$, estrogen $(\mathrm{P}=0.0480)$ and combined treatment $(\mathrm{P}=0.0032)$ (Fig. 3) compared with the IUA model group. The expression of vimentin showed no significant difference among the groups (Fig. 3). In contrast with the control group, the expression levels of RhoA $(\mathrm{P}=0.0243)$, RhoB $(\mathrm{P}=0.0044)$ and $\mathrm{RhoC}(\mathrm{P}=0.0463)$ were significantly 
A
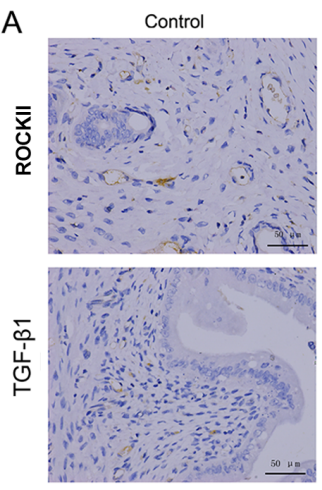

B

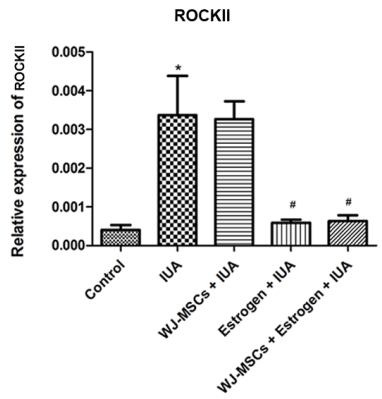

WJ-MSCs + IUA
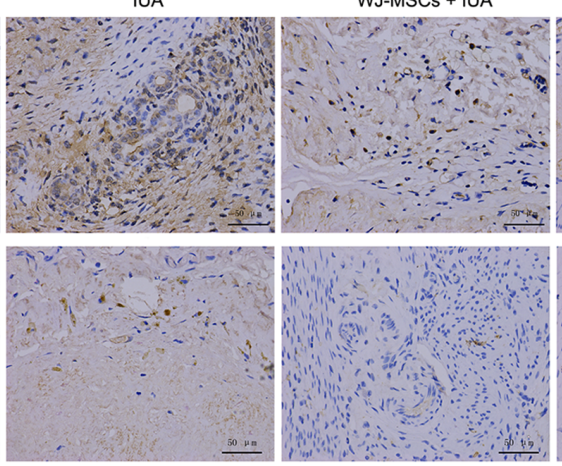

Estrogen + IUA

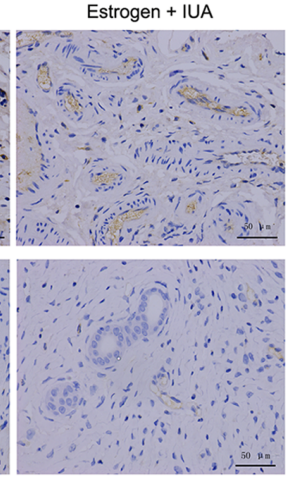

WJ-MSCs + Estrogen + IUA
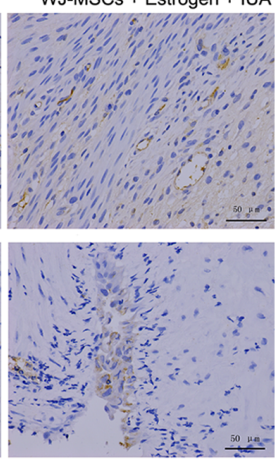

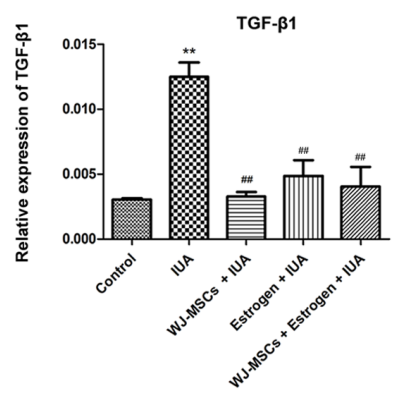

Figure 6. (A) Immunohistochemical staining, and (B) semi-quantitative analysis of immunohistochemical data for ROCKII and TGF- $\beta 1$ in each group. Scale bar, $50 \mu \mathrm{m}$. ${ }^{*} \mathrm{P}<0.05,{ }^{* *} \mathrm{P}<0.01$ compared with the control group; ${ }^{*} \mathrm{P}<0.05,{ }^{\# \#} \mathrm{P}<0.01$ compared with the model group. IUA, intrauterine adhesion; ROCKII, Rho-associated coiled-coil-containing protein kinase II; TGF- $\beta 1$, transforming growth factor- $\beta 1$; WJ-MSCs, Wharton's jelly mesenchymal stem cells.

higher in the IUA model group (Figs. 4 and 5). However, their expression levels varied after different treatment interventions. As compared with the IUA model group, the expression of RhoA was significantly decreased in WJ-MSCs $(\mathrm{P}=0.0248)$ and WJ-MSCs + estrogen treatment groups $(\mathrm{P}=0.0247)$, but was not significantly changed $(\mathrm{P}=0.4299)$ after estrogen treatment alone (Fig. 4); however, the expression of RhoB was only significantly decreased $(\mathrm{P}=0.0004)$ after WJ-MSCs + estrogen treatment (Fig. 4) and the expression of RhoC was not obviously changed in all treatment groups (Fig. 5). In addition, the expression levels of ROCKI ( $\mathrm{P}=0.0080$; Fig. 5), ROCKII ( $\mathrm{P}=0.0446$; Fig. 6) and TGF- $\beta 1$ ( $\mathrm{P}=0.0013$; Fig. 6$)$ were significantly increased in the IUA model group compared with the control group. Notably, the expression level changes in ROCKI and TGF- $\beta 1$ were similar in all treatment groups; these markers were significantly downregulated in WJ-MSCs (ROCKI, $\mathrm{P}=0.0002$; TGF- $\beta 1$, $\mathrm{P}=0.0013)$, estrogen (ROCKI, $\mathrm{P}=0.0012$; TGF- $\beta 1, \mathrm{P}=0.0045)$ and WJ-MSCs + estrogen treatment groups (ROCKI, $\mathrm{P}=0.0008$; TGF- $\beta 1, P=0.0020)$ compared with the IUA model group. The expression of ROCKII was significantly reduced in estrogen $(\mathrm{P}=0.0204)$ and $\mathrm{WJ}-\mathrm{MSCs}+$ estrogen treatment groups $(\mathrm{P}=0.0222)$ compared with that in the IUA model group, but no significant difference was detected between the model group and WJ-MSCs treatment group $(\mathrm{P}=0.9998)$.

\section{Discussion}

In previous years, studies have revealed that the occurrence of IUA may be associated with decrease or loss of endometrial stem cells, thus utilizing stem cell transplantation as a method of treating IUA has been proposed $(22,23)$. In the present study, intrauterine administration of WJ-MSCs and estrogen, separately, or combined to female IUA rats with a thin endometrium was conducted. The H\&E results showed that IUA symptoms were alleviated to some degree in all treatment groups, but the degree of restoration was better in the independent WJ-MSCs and estrogen treatment groups than in the combined treatment group. The immunohistochemistry analysis revealed that pan-keratin expression was increased, whereas RhoA, ROCKI and TGF- $\beta 1$ expression was decreased in IUA rats in the WJ-MSCs treatment group; however, no significant change in the expression levels of these proteins was observed among the three treatment groups.

Keratins encode cytoskeletal polymers and are members of the intermediate filament protein family (24). Keratins are differentially expressed in various epithelial cells at different stages of development and differentiation, thus specific keratins may serve as markers to distinguish different epithelial cell types (25). Mcintosh et al (26) used a pan-keratin antibody to detect the multiple types of keratin present in cervical epithelium. In the present study, pan-keratin was selected as a marker of endometrial epithelial cells, and a significant decrease in keratin expression was detected in IUA rats compared with in normal rats. Consistent with these results, the expression of keratin in IUA rats was significantly reduced compared with control rats in a previous study (27). After WJ-MSCs treatment, the enhanced expression of keratin indicated that WJ-MSCs may have differentiated into endometrial epithelial cells.

Epithelial fibrosis, where the stroma is largely replaced with fibrous tissue, is the main manifestation of IUA (28). TGF- $\beta$, which is a multifunctional cytokine, is a powerful inducer of the extracellular matrix (ECM) (29). Tissue fibrosis is a result of exacerbated ECM deposition, and activated TGF- $\beta 1$ can promote collagen deposition by regulating ECM gene expression (30). Several studies have demonstrated that overexpression of TGF- $\beta$ was involved in IUA forma- 
tion $(31,32)$, which is consistent with the present results. Notably, a significant decrease in TGF- $\beta$ was detected in IUA rats after WJ-MSCs treatment, which indicated that WJ-MSCs intervention might alleviate epithelial fibrosis in IUA rats via regulating TGF- $\beta 1$.

Notably, TGF- $\beta 1$ is an upstream signal factor of Rho, which is one of the most important members of the Rho GTPase family, which includes A, B and C isoforms (14). ROCK is the most studied downstream effector molecule of Rho (15). It has been reported that the TGF- $\beta 1$-mediated Rho/ ROCK signaling pathway may participate in lung fibroblast transformation (33) and TGF- $\beta 1$-induced ROCK elevation could strengthen the fibrotic response (34). In addition, TGF- $\beta$ may promote ECM components, such as fibronectin, laminin, MMP-2 and type I collagen via activating the RhoA/ROCK signaling pathway (35). However, whether the Rho/ROCK signaling pathway mediated by TGF- $\beta 1$ is associated with epithelial fibrosis of IUA remains unclear. Therefore, this study detected the expression of TGF- $\beta 1$, RhoA, RhoB, RhoC, ROCKI and ROCKII in each group. As a result, the expression levels of TGF- $\beta 1$, RhoA and ROCKI were all significantly increased in IUA rats and reduced after WJ-MSCs intervention. Conversely, RhoB, RhoC and ROCKII expression were not obviously changed after WJ-MSCs intervention. Thus, it may be hypothesized that WJ-MSCs intervention reduces epithelial fibrosis of IUA rats via inhibition of the RhoA/ ROCKI signaling pathway, induced by TGF- $\beta 1$.

Estrogen is secreted by ovaries and is an important steroid hormone for endometrial repair; it promotes cell mitosis of endometrial epithelium and stromal cells (36). Reportedly, estrogen may also be essential for endometrial hyperplasia and differentiation $(37,38)$. The role of estrogen in endometrial fibrosis cannot be ignored. The different dosages of estrogen have been reported to serve different effects on endometrial fibrosis and in the endometrium by regulating the expression of TGF- $\beta 1$ (39). Notably, estrogen has been reported to suppress the progression of fibrosis in myofibroblasts with decreased collagen expression by inhibiting TGF- $\beta 1$ and the Rho/ROCK signaling pathways (40). In addition, estradiol attenuated myocardial fibrosis by suppressing the RhoA/ ROCK/cofilin pathway in ovariectomized female infarcted rats $(41,42)$. Similarly, in the present study, the expression levels of TGF- $\beta 1$ and RhoA/ROCKI were inhibited in IUA model rats after WJ-MSCs + estrogen treatment. However, the therapeutic effect was not improved by a combined therapy of WJ-MSCs + estrogen compared with WJ-MSCs or estrogen separately. Thus, it was hypothesized that estrogen might not directly affect the differentiation of WJ-MSCs into endometrial epithelial cells to alleviate endometrial fibrosis. Further experiments are required to determine the underlying mechanism of estrogen treatment, and to determine if the two mechanisms act independently or if they are interconnected. In addition, almost all experimental designs focused on the structure of uterine tissue. It is not clear if the organic function can also be restored by combined WJ-MSCs + estrogen treatment. Moreover, it is important to verify whether WJ-MSCs injection delivered enough stem cells in the uterine tissue, and if WJ-MSCs differentiated into endometrial epithelial cells by relevant experiments. These will need to be investigated in the future.
In conclusion, the present study indicated that treatment with WJ-MSCs, independently or as a combined therapy with estrogen, may reverse epithelial fibrosis in IUA rats. Inhibition of the RhoA/ROCKI signaling pathway induced by TGF- $\beta 1$ may be involved in the treatment. Notably, the endometrial restoration in IUA rats was better following WJ-MSCs intervention alone compared with the combined therapy.

\section{Acknowledgements}

Not applicable.

\section{Funding}

No funding was received.

\section{Availability of data and materials}

The datasets used and/or analyzed during the current study are available from the corresponding author on reasonable request.

\section{Authors' contributions}

$\mathrm{ZH}$ conceived and designed the research, and revised the manuscript for important intellectual content. YZ, LD, JL and $\mathrm{BH}$ acquired, analyzed and interpreted data, conducted statistical analysis and drafted the manuscript. All authors read and approved the final manuscript.

\section{Ethics approval and consent to participate}

The experimental protocol used in this study was approved by the Animal Care and Use Committee of the People's Hospital of Wuhan University.

\section{Patient consent for publication}

Not applicable.

\section{Competing interests}

The authors declare that they have no competing interests.

\section{References}

1. Berman JM: Intrauterine adhesions. Semin Reprod Med 26: 349-355, 2008.

2. Deans R and Abbott J: Review of intrauterine adhesions. J Minim Invasive Gynecol 17: 555-569, 2010.

3. Luo S, Shen L and Zhang X: Clearing heat and activating blood method for intrauterine adhesion. J Changchun Univ Tradit Chin Med 1: 78-80, 2016.

4. Panayotidis C, Weyers S, Bosteels $\mathrm{J}$ and Herendael BV: Intrauterine adhesions (IUA): Has there been progress in understanding and treatment over the last 20 years? Gynecol Surg 6: 197-211, 2009

5. Johary J, Xue M, Zhu X, Xu D and Velu PP: Efficacy of estrogen therapy in patients with intrauterine adhesions: Systematic review. J Minim Invasive Gynecol 21: 44-54, 2014.

6. Ebrahim N, Mostafa O, El Dosoky RE, Ahmed IA, Saad AS, Mostafa A, Sabry D, Ibrahim KA and Farid AS: Human mesenchymal stem cell-derived extracellular vesicles/estrogen combined therapy safely ameliorates experimentally induced intrauterine adhesions in a female rat model. Stem Cell Res Ther 9: 175, 2018.

7. Gargett $\mathrm{CE}$ and Ye L: Endometrial reconstruction from stem cells. Fertil Steril 98: 11-20, 2012. 
8. Fong CY, Chak LL, Biswas A, Tan JH, Gauthaman K, Chan WK and Bongso A: Human Wharton's jelly stem cells have unique transcriptome profiles compared to human embryonic stem cells and other mesenchymal stem cells. Stem Cell Rev Rep 7: 1-16, 2011.

9. Taghizadeh RR, Cetrulo KJ and Cetrulo CL: Wharton's Jelly stem cells: Future clinical applications. Placenta 32 (Suppl 4): S311-S315, 2011.

10. Chen X, Yang X, Wu R, Chen W, Xie H, Qian X and Zhang Y: Therapeutic effects of Wharton jelly-derived mesenchymal stem cells on rat abortion models. J Obstet Gynaecol Res 42: 972-982, 2016.

11. Yu D, Wong YM, Cheong Y, Xia E and Li TC: Asherman syndrome - one century later. Fertil Steril 89: 759-779, 2008.

12. Li J, Du S, Sheng X, Liu J, Cen B, Huang F and He Y: MicroRNA-29b inhibits endometrial fibrosis by regulating the Sp1-TGF- $\beta 1 /$ Smad-CTGF Axis in a Rat Model. Reprod Sci 23 386-394, 2016.

13. Liu X, Duan H, Zhang HH, Gan L and Xu Q: Integrated Data Set of microRNAs and mRNAs Involved in Severe Intrauterine Adhesion. Reprod Sci 23: 1340-1347, 2016.

14. Defert $O$ and Boland S: Rho kinase inhibitors: A patent review (2014-2016). Expert Opin Ther Pat 27: 507-515, 2017.

15. Ji H, Tang H, Lin H, Mao J, Gao L, Liu J and Wu T: Rho/Rock cross-talks with transforming growth factor- $\beta /$ Smad pathway participates in lung fibroblast-myofibroblast differentiation. Biomed Rep 2: 787-792, 2014.

16. Kohno M, Watanabe M, Goto T, et al. Attenuation Lung Ischemia-Reperfusion Inj Rho-Associated Kinase Inhib Rat Model Lung Transplant 20: 359-364, 2014.

17. Ivaska J, Pallari H-M, Nevo J and Eriksson JE: Novel functions of vimentin in cell adhesion, migration, and signaling. Exp Cell Res 313: 2050-2062, 2007.

18. Magin TM, Vijayaraj $P$ and Leube RE: Structural and regulatory functions of keratins. Exp Cell Res 313: 2021-2032, 2007.

19. Clark JD, Gebhart GF, Gonder JC, Keeling ME and Kohn DF: Special Report: The 1996 Guide for the Care and Use of Laboratory Animals. ILAR J 38: 41-48, 1997.

20. Ren H, Sang Y, Zhang F, Liu Z, Qi N and Chen Y: Comparative analysis of human mesenchymal stem cells from umbilical cord dental pulp, and menstrual blood as sources for cell therapy. Stem Cells Int 2016: 3516574, 2016.

21. Zhang L, Li Y, Guan CY, Tian S, Lv XD, Li JH, Ma X and Xia HF: Therapeutic effect of human umbilical cord-derived mesenchymal stem cells on injured rat endometrium during its chronic phase. Stem Cell Res Ther 9: 36, 2018.

22. Wang J, Ju B, Pan C, Gu Y, Zhang Y, Sun L, Zhang B and Zhang Y: Application of Bone Marrow-Derived Mesenchymal Stem Cells in the Treatment of Intrauterine Adhesions in Rats. Cell Physiol Biochem 39: 1553-1560, 2016.

23. Zheng SX, Wang J, Wang XL, Ali A, Wu LM and Liu YS: Feasibility analysis of treating severe intrauterine adhesions by transplanting menstrual blood-derived stem cells. Int J Mol Med 41: 2201-2212, 2018

24. Pan X: Regulation of skin keratin intermediate filament proteins by phosphorylation. Dissertations \& Theses - Gradworks, 2012.

25. Fuchs E and Weber K: Intermediate filaments: Structure, dynamics, function, and disease. Annu Rev Biochem 63: 345-382, 1994.

26. McIntosh PB, Laskey P, Sullivan K, Davy C, Wang Q, Jackson DJ, Griffin HM and Doorbar J: E1--E4-mediated keratin phosphorylation and ubiquitylation: A mechanism for keratin depletion in HPV16-infected epithelium. J Cell Sci 123: 2810-2822, 2010.
27. Zhou Q, Wu X, Hu J and Yuan R: Abnormal expression of fibrosis markers, estrogen receptor $\alpha$ and stromal derived factor 1/chemokine (C-X-C motif) receptor 4 axis in intrauterine adhesions. Int J Mol Med 42: 81-90, 2018.

28. Zhu HY, Ge TX, Pan YB and Zhang SY: Advanced role of hippo signaling in endometrial fibrosis: Implications for intrauterine adhesion. Chin Med J (Engl) 130: 2732-2737, 2017.

29. Guarino M, Tosoni A and Nebuloni M: Direct contribution of epithelium to organ fibrosis: Epithelial-mesenchymal transition. Hum Pathol 40: 1365-1376, 2009.

30. Verrecchia $\mathrm{F}$ and Mauviel A: Transforming growth factor-beta and fibrosis. World J Gastroenterol 13: 3056-3062, 2007.

31. Salma U, Xue M, Ali Sheikh MS, Guan X, Xu B, Zhang A, Huang $\mathrm{L}$ and $\mathrm{Xu} \mathrm{D}$ : Role of transforming frowth factor- $\beta 1$ and smads signaling pathway in intrauterine adhesion. Mediators Inflamm 2016: 4158287, 2016.

32. Xue X, Chen Q, Zhao G, Zhao JY, Duan Z and Zheng PS: The Overexpression of TGF- $\beta$ and $\mathrm{CCN} 2$ in Intrauterine Adhesions Involves the NF-кB Signaling Pathway. PLoS One 10: e0146159, 2015.

33. Ji H, Tang H, Lin H, Mao J, Gao L, Liu J and Wu T: Rho/Rock cross-talks with transforming growth factor- $\beta /$ Smad pathway participates in lung fibroblast-myofibroblast differentiation. Biomed Rep 2: 787-792, 2014.

34. Wang S, Sun A, Li L, Zhao G, Jia J, Wang K, Ge J and Zou Y: Up-regulation of BMP-2 antagonizes TGF- $\beta 1 /$ ROCK-enhanced cardiac fibrotic signalling through activation of Smurf1/Smad6 complex. J Cell Mol Med 16: 2301-2310, 2012.

35. Zhu J, Nguyen D, Ouyang H, Zhang XH, Chen XM and Zhang K: Inhibition of RhoA/Rho-kinase pathway suppresses the expression of extracellular matrix induced by CTGF or TGF- $\beta$ in ARPE-19. Int J Ophthalmol 6: 8-14, 2013.

36. Adesanya OO, Zhou J, Samathanam C, Powell-Braxton L and Bondy CA: Insulin-like growth factor 1 is required for G2 progression in the estradiol-induced mitotic cycle. Proc Natl Acad Sci USA 96: 3287-3291, 1999.

37. Hu K, Zhong G and He F: Expression of estrogen receptors ERalpha and ERbeta in endometrial hyperplasia and adenocarcinoma. Int J Gynecol Cancer 15: 537-541, 2005.

38. Osteen KG and Anderson TL: Effect of estrogen on human endometrial epithelial cell growth and differentiation in vitro. Steroids 56: 279-283, 1991.

39. Zhou Q, Wu X, Dai X, Rui Y and Qi H: The different dosages of estrogen affect endometrial fibrosis and receptivity, but not SDF-1/CXCR4 axis in the treatment of intrauterine adhesions. Gynecol Endocrinol 34: 49-55, 2018.

40. Jiang $H$ and Dai Y: Estrogen attenuates the TGF $\beta 1$-induced conversion of primary penile tunica albuginea fibroblasts into myofibroblasts and inhibits collagen production and myofibroblast contraction by modulating the Smad and Rho/ROCK signaling pathways. Transl Androl Urol 4: 463-472, 2015.

41. Luft FC: ER $\alpha$ on the cell membrane helps the heart. J Mol Med (Berl) 92: 1-3, 2014.

42. Lee TM, Lin SZ and Chang NC: Membrane ER $\alpha$ attenuates myocardial fibrosis via RhoA/ROCK-mediated actin remodeling in ovariectomized female infarcted rats. J Mol Med (Berl) 92: 43-51, 2014.

This work is licensed under a Creative Commons Attribution-NonCommercial-NoDerivatives 4.0 International (CC BY-NC-ND 4.0) License. 